\section{“SER DEVORADO NO DUELE”. THEODOR W. ADORNO Y LA EXPERIENCIA AMERICANA}

\author{
Jordi Maiso \\ Universidad de Salamanca \\ jordimaiso@hotmail.com
}

\section{DOES NOT HURT TO BE DEVOURED. TH. W. ADORNO AND THE AMERICAN EXPERIENCE}

\begin{abstract}
The present text aims to fathom Theodor W. Adorno's american experience as a deciding learning process in the development of his Critical Theory. As a consequence of his New York exile years and his participance on the Princeton Radio Research Project, Adorno not only gets in touch with the proceeding of the culture industry and sets down a resolute theoretical approach to radio music, but also realizes a shift has taken place on the social function of theory which enables him a poignant self-reflection on the situation of the emigrated intellectual.
\end{abstract}

KEY WORDS: Theodor W. Adorno; exile; radio music; administrative research; Critical Theory; culture industry; social condition of the intellectual; Advanced Capitalism.

"Ich wäre gerne auch weise. In den alten Bücher steht, was weise ist: sich aus dem Streit der Welt halten und die kurze Zeit ohne Furcht verbringen"

(Bertolt Brecht, An die Nachgeborenen)

Entre los intelectuales alemanes exiliados para los que la experiencia del desajuste entre Europa y América se convierte en una fuente de conocimiento, Theodor W. Adorno ocupa un lugar de excepción. Textos como Minima moralia o Dialéctica de la llustración, convertidos en clásicos para varias generaciones de lectores, no sólo fueron redactados en el exilio americano, sino que revelan un poso de experiencias biográficas y profesionales que el francfortiano fue capaz de sublimar en teoría. El conocimiento surgido de la elaboración de lo vivido durante estos años alcanza tanto a la realidad europea que los emigrados se habían visto obligados a abandonar como a la América que se convirtió en tierra de acogida y, sobre todo, se constituye como autorreflexión sobre la figura del
RESUMEN: El presente texto pretende desentrañar la experiencia americana de Theodor $W$. Adorno como un proceso de aprendizaje decisivo en la formulación de su Teoria Crítica. A partir de su exilio en Nueva York y de su participación en el Princeton Radio Research Project, Adorno no sólo adquiere un conocimiento directo del funcionamiento de la industria de la cultura y formula una decisiva aproximación teórica a la música radiofónica, sino que también toma conciencia de un cambio en la función social de la teoria que le permite llevar a cabo una aguda autorreflexión sobre la situación del intelectual emigrado.

PALABRAS CLAVE: Theodor W. Adorno; exilio; música radiofónica; administrative research; Teoría Crítica; industria de la cultura; condición social del intelectual; capitalismo avanzado.

intelectual europeo exiliado en Estados Unidos. El propio Adorno fue muy consciente de la transformación que la experiencia del exilio ejercería en su propia trayectoria intelectual, como revela el hecho de que, al final de su vida, con el movimiento estudiantil alemán en plena ebullición y encontrándose en el apogeo de su producción teórica, sacara tiempo para elaborar sus recuerdos en un texto - por lo demás no exento de cierta estilización autobiográfica- como "Experiencias científicas en Estados Unidos". Pero sus reflexiones sobre la condición del exiliado se reparten por toda su obra, alcanzando su formulación más contundente en algunos de sus escritos considerados menores. No en vano Adorno se cuenta entre los autores que nos ha legado de forma más indeleble el significado del exilio estadounidense para un intelectual europeo. Sin embargo, al mismo tiempo, resulta obligatorio constatar que el consenso académico acerca de lo paradigmático de su figura como exiliado ha acabado por recortar el alcance de su testimonio. 
En efecto, a partir de la muerte de Adorno, la literatura secundaria ha tendido a presentar sus textos producidos en Estados Unidos como el testimonio de un mundo que estaba desapareciendo de manera irremisible y para siempre: el del intelectual europeo de formación burguesa que sucumbia ante la incipiente industria de la cultura. Desde esta perspectiva, el pensamiento adorniano aparecería teñido de un resentimiento elitista con respecto a la cultura de masas americana que lo revelaría anticuado e inservible para toda apropiación contemporánea. Del mismo modo, la concepción de la Teoría Crítica como un planteamiento que, al carecer de un destinatario definido en su momento histórico, se entiende a sí misma como mensaje en una botella, cobraría un nuevo significado. Ya no se entendería como un análisis que busca un sujeto capaz de una transformación que en dicha situación histórica nadie parece en condiciones de llevar a cabo, sino que sería simplemente el testimonio del naufragio histórico de la antigua alta cultura. En el presente texto quisiera contribuir a corregir esta imagen apoyándome en materiales e investigaciones recientemente publicadas'. El punto de partida para ello será el concepto adorniano de experiencia americana, que remite a la estrecha interrelación entre la sociedad capitalista más avanzada, en la que nada queda fuera de su tupida red de socialización, y la transformación funcional del individuo y la teoría en ella. De hecho, el concepto de Teoría Crítica como tal pierde su especificidad si se abstrae de las experiencias del exilio americano (Claussen, 1999, 27 y ss.). En consecuencia, el elemento fundamental para entender lo que el exilio aportó al pensamiento adorniano habrá de buscarse en su registro de las constantes tensiones entre el imperativo de adaptación y la voluntad de preservación de la propia identidad, entre la inserción del intelectual en un marco académico profesionalizado y la defensa de la autonomía del pensamiento $y$, en último término, en la insistencia en que, aunque no sea más que como un reducto no asimilable a las concentraciones de poder, el intelectual y la teoria no renuncien al elemento de resistencia que su exigencia de verdad supone en una sociedad que busca poner todo el progreso técnico-científico al servicio de la maximización del beneficio. De esta manera el concepto adorniano de experiencia americana puede también rebasar su propio núcleo temporal y conseguir interpelarnos, poniendo en duda seguridades que, un tanto ingenuamente, algunos seguimos dando por sentadas.

Retrospectivamente, Adorno ha reconocido que, desde el primero hasta el último día de su exilio americano, se consi- deró un europeo, y que en todo momento trató de aferrarse a una cierta "continuidad espiritual" que le permitiera no romper del todo con su biografía personal e intelectual anterior. Ha de tenerse en cuenta que esto tiene lugar en un momento en el que "adjustment era todavía una palabra mágica, sobre todo respecto a quien habia tenido que huir de Europa en calidad de perseguido, y del que se esperaba tanto que se cualificara profesionalmente en el nuevo pais como que no se anquilosara con soberbia en lo que una vez había sido" (Adorno, 2003a, 702). Frente a ello, el francfortiano insistió en todo momento en que toda conciencia política de los exiliados partía de la imposibilidad de considerarse inmigrantes que llegaban a Estados Unidos a probar fortuna, sino que eran ante todo emigrantes que, por haber sufrido la persecución en su propio país, se habian visto obligados a buscar cobijo al otro lado del Atlántico (Adorno, 2003b, 352). Resulta evidente que el exiliado que no quisiera renunciar a cierta continuidad biográfica y de intereses en una sociedad que no ofrecia más alternativas que la integración total en su mercado laboral, se vería obligado a pagar el precio de la permanente condición de outsider, de un constante permanecer extraño a cuanto le rodea. Y sin embargo la imagen de un Adorno desvinculado de la cotidianeidad cultural, académica y mediática americana, así como el mito de que la crítica adorniana a la cultura estadounidense está formulada desde la desdeñosa distancia de un europeo elitista que desconoce la realidad a la que no ha logrado adaptarse, son rigurosamente falsos. Al fin y al cabo, el francfortiano no sólo vivió quince años en Estados Unidos, sino que llegó a ser, durante más de una década, ciudadano americano. Por lo demás Adorno tuvo ocasión de conocer de primera mano el panorama científico y de investigación, así como la industria radiofónica y Hollywood, y sobre todo se convirtió en un agudo observador que aportó alguna de las más lúcidas reflexiones sobre la condición misma del exiliado.

Por tanto, durante sus años en Estados Unidos, Adorno consiguió convertir su extrañeza con respecto a la sociedad americana en una productiva fuente de conocimiento. Muchos de sus textos buscan dar cuenta de la experiencia del teórico europeo que, forzado a emigrar, se topa con la realidad cultural, científica y académica estadounidense, con la lucha por la supervivencia en el mercado del conocimiento y con el imperativo de la adaptación, pero probablemente pocos pasajes logran plasmar esta experiencia de manera tan elocuente como uno de los sueños recogido 
en sus Traumprotokolle. Fechada en Los Ángeles en enero de 1942, apenas unos meses después de haber abandonado Nueva York, la transcripción del sueño nos relata cómo, en un paseo por Fráncfort, Adorno acaba casualmente en la oficina privada del presidente Roosevelt. El intelectual exiliado topa por azar con el alto núcleo del poder político $y$, una vez alli, el presidente, paternal, le permite desatender el protocolo y tomar un libro de su biblioteca. Cuando William S. Knudsen, en aquella época el presidente prohitleriano de General Motors, entra en el despacho para hablar con Roosevelt de cuestiones de seguridad nacional, Adorno es cordialmente invitado a abandonar la sala. Una vez fuera de la habitación, al salir del ascensor, no halla un acceso que le devuelva a las calles de Fráncfort, sino que se encuentra en un pozo subterráneo y busca desesperado una elevación para salvarse. Sin embargo, antes de poder alcanzar la sali$\mathrm{da}$, se ve rodeado por unos cocodrilos tan seductores como amenazantes, con cuya descripción finaliza la transcripción del sueño: "Tenían las cabezas de mujeres extraordinariamente hermosas. Una se dirigió a mí. Ser devorado no duele. Para hacérmelo más fácil, me prometió antes las cosas más hermosas" (Adorno, 2005, 14).

En este sueño se plasma admirablemente la dialéctica entre preservación de la propia identidad y la necesidad de adjustment que presidía la vida de todo exiliado. En las siguientes páginas intentaré desentrañar las experiencias del exilio que hicieron a Adorno consciente de las implicaciones del modelo social del capitalismo avanzado y de las transformaciones de la función del individuo, del intelectual y de la teoria aparejadas a éste, que subyacen a las tensiones que emergen -de forma inusitadamente explícita- en la transcripción de esta pesadilla. Para ello me centraré en los primeros años de Adorno en Estados Unidos, concretamente en el período que pasa en Nueva York entre febrero de 1938 y 1941, en el que su ingenuidad con respecto a la realidad americana es más explícita y su choque con ella más contundente.

\section{LA EMIGRACIÓN INTELECTUAL Y EL INSTITUTO DE INVESTIGACIÓN SOCIAL}

A lo largo de la década de 1930, especialmente en los años del New Deal, el gobierno estadounidense siguió una política de emigración relativamente liberal, que permitió a los europeos amenazados por el régimen nacional-socialista y su progresiva expansión encontrar un refugio al otro lado del Atlántico. En principio, Estados Unidos parecía un destino idóneo para los exiliados, ya que, pese a la persistencia de las consecuencias de la Gran Depresión, estaba menos encasillado en tradiciones y tenía una estructura que permitía la coexistencia de diferentes minorías. No en vano Nueva York era el centro de los intelectuales emigrados. Sin embargo la sociedad norteamericana tenía otras exigencias para quienes quisieran integrarse en ella. En los escritos de Adorno, Estados Unidos es caracterizado como un "país radicalmente burgués" (Adorno, 2003c, 310), que representaba el estadio más avanzado en la evolución de la sociedad industrializada, y ello iba a suponer también un choque importante para muchos exiliados. La expansión del fascismo en Europa había dado lugar a un nuevo fenómeno migratorio en el que el objetivo de los europeos que ponían rumbo al "pais de las posibilidades ilimitadas" ya no era, como en la segunda mitad del XIX, hacer fortuna y alcanzar una cota de bienestar que se les negaba en sus paises de origen, sino simplemente salvar su vida. Las universidades alemanas habian sido limpiadas de judíos, homosexuales, comunistas y socialdemócratas y, para 1938, el 45 por 100 de las plazas de funcionarios científicos alemanes tenían nuevos ocupantes (Erdmann, 1989, 171). Pero la persecución no se había detenido en la purga de la academia, y los profesionales universitarios eran los menos de los intelectuales alemanes obligados al exilio. En un texto de 1942 el propio Adorno se muestra consciente de lo específico de esta "emigración intelectual", que habia llevado a desembarcar en Estados Unidos a

"la intelligentsia europea como capa, y no sólo los judíos. Lo que pretendian no era vivir mejor, sino sobrevivir; las posibilidades del nuevo mundo habian dejado de ser ilimitadas hacia ya tiempo, por lo cual el Diktat de la adaptación se impuso a los intelectuales mismos después de triunfar plenamente en el terreno de la competencia económica. En lugar de la selva en la que el pionero -también el de espíritu- espera regenerarse, hay ya una civilización que, como sistema, aferra la vida entera sin dejar siquiera a la conciencia de los emigrantes los escondrijos que la negligencia europea mantuvo abiertos en la época de los grandes trusts" (Adorno, 1962, 99-100).

Lo que los exiliados se encuentran al llegar a Estados Unidos es un país regido de principio a fin por el principio 
de rentabilidad económica: "Lo que no se registra aquí y ahora como socialmente provechoso en el mercado, no vale y será olvidado" (Adorno, 2003c, 311). Esto imposibilitaba toda conciencia de continuidad temporal con la vida anterior al otro lado del Atlántico pero, sobre todo, Adorno advierte que, cuando semejante imperativo se ejerce sobre la emigración intelectual, sucede algo cualitativamente distinto de lo que ocurria cuando se exigía a quienes llegaban al pais en busca de fortuna. Lo que distingue al intelectual de aquellos que están sujetos a las exigencias de la reproducción material de la vida es su capacidad de "distanciarse intelectualmente del poder superior de la furiosa existencia cotidiana" (Adorno, 1962, 99). Con esto no se pretende dispensar la miseria de quienes no se hallan en semejante privilegiada posición, sino señalar el elemento novedoso del capitalismo avanzado norteamericano, en el que se exige a los intelectuales renunciar a su independencia y a su autonomía: "En el dominio económico todo se ajusta convenientemente según las leyes de la oferta y la demanda. Que se extiendan al espíritu, que éste acabe siendo absorbido por el complejo funcional, forma parte de la lógica del sistema, pero al mismo tiempo contradice el principio mismo del espíritu, el cual no debe agotarse en la reproducción de la vida y creando consciencia de lo existente perfila en negativo algo distinto posible" (Adorno, 2003d, 387-388). Con ello tendía a reducirse también la calidad del propio trabajo intelectual, ya que "Entre la reproducción de la propia vida bajo el monopolio de la cultura de masas y el trabajo objetivo y responsable existía una ruptura irreconciliable" (Adorno, 2003e, 35). Lo que la experiencia americana revela al hombre de teoria es que el conocimiento mismo se ha convertido en mercancía, con lo cual el intelectual se hace consciente de su impotencia con respecto a la maquinaria socioeconómica y reacciona con pánico. Su fuerza de trabajo ha perdido su posición privilegiada y ahora su posición es de aislamiento y abandono a la suerte de la competencia mercantil en la industria del conocimiento. Las condiciones materiales en que la mayoría se vio forzada a exiliarse, tal y como las relata el propio Adorno en un texto dedicado a Sigfried Kracauer con motivo de su 75 cumpleaños, completan el cuadro de la menesterosa situación del intelectual emigrado:

"Las reglamentaciones sobre divisas y los impuestos especiales obligaron a los intelectuales a expatriarse literalmente como mendigos. El cálculo de los nacionalsocialistas de que con ello aquellos a quien ellos odiaban tampoco serian bien vistos alli donde encontraran refugio no andaba del todo errado. El hecho de que no pocos Estados sólo aceptaran a quienes contaban con destrezas prácticas útiles arroja incluso luz sobre países que renunciaron a semejantes alambradas de púas. En todas partes el intelectual, en cuanto no hubiera mostrado su capacitación en el seno de la comunidad científica establecida mediante trabajos Ilamados positivos o al menos procediera de la jerarquía universitaria, se sentía superfluo. Probablemente, la compulsión a integrarse era peor que en anteriores emigraciones. En la mayoría de paises de acogida la red social era demasiado espesa, el thought control demasiado riguroso. La amenaza de paro hacia indeseables a los potenciales competidores" (Adorno, 2003d, 387).

Lo que Adorno estiliza en este pasaje como "indeseabilidad" de los "potenciales competidores", fue en realidad un ambiente de terrible competencia entre los intelectuales exiliados, en una desesperada lucha por la supervivencia material que estaria dominada por rivalidades, envidias y desconfianzas. Probablemente a la luz de estas circunstancias cobra también otro significado la célebre afirmación adorniana de que "Todo intelectual en el exilio, sin excepción, lleva una vida dañada, y hace bien en reconocerlo él mismo si no quiere ser cruelmente informado de ello tras las puertas herméticamente cerradas de su autoestima" (Adorno, 2003e, 35). La competencia entre los emigrados fue especialmente despiadada cuando se luchaba por ganar el favor de instituciones o núcleos de poder que podían ofrecer una cierta estabilidad, como era el caso del Instituto de Investigación Social dirigido por Max Horkheimer, que durante los primeros años del exilio gozó de una ventajosa afiliación a la neoyorquina Universidad de Columbia ${ }^{2}$.

Sin lugar a dudas, el Instituto de Investigación Social, que había llegado a Nueva York en 1934 tras un breve paso por Ginebra ${ }^{3}$, jugó un papel decisivo para muchos emigrados. En primer lugar aseguró la vida de sus colaboradores más íntimos durante los años más difíciles y les ofreció una estructura institucional que les permitió trabajar sin necesidad de ajustarse a las obligaciones de la carrera académica. Sólo gracias a esta estructura organizativa cuidada por Horkheimer y Pollock en los años del exilio pudo tomar forma el proyecto de la Teoría Crítica. Pero el Instituto también prestó un apoyo decisivo a la comunidad de exiliados alemanes en Europa y América, consiguiendo 
visados que permitieron a algunos escapar de la catástrofe europea y asignando sueldos que posibilitaron a varios de sus colaboradores continuar con sus investigaciones con una cierta independencia de la rentabilización inmediata exigida en el mercado del conocimiento. Entre los que, de un modo u otro, se beneficiaron de su ayuda, se cuentan los nombres de Karl Korsch, Walter Benjamin, Ernst Bloch, Sigfried Kracauer o Ludwig Marcuse. Por su parte, la Zeitschrift für Sozialforschung publicada por el Instituto supuso también un órgano importante para la comunidad de emigrados. Centrada en el análisis de los problemas más acuciantes para quienes se habían visto obligados a abandonar Europa (autoridad, prejuicio, cultura de masas o relación entre capitalismo y fascismo), ofreció a la intelectualidad exiliada un material de debate y reflexión de alto nivel teórico y contribuyó a paliar el aislamiento de muchos emigrados. Sin embargo, al mismo tiempo, la posibilidad de estabilidad que ofrecía el Instituto a quienes lograran convertirse en sus colaboradores le situó en el centro de un penoso cuadro de lucha por la autoconservación como mera autoconservación que su dirección no siempre gestionó de manera ejemplar. En una sociedad absolutamente jerarquizada como la americana, al intelectual aislado en el exilio no le quedaba otra posibilidad que aproximarse a posiciones de poder e influencia, y Horkheimer atesoraba ambas. De hecho su situación como director del Instituto y manager intelectual también dio lugar a una feroz competencia entre los propios miembros del Instituto. Todos anhelaban ganarse su favor para garantizar su estabilidad y asegurarse un trabajo teórico libre de presiones externas, pero sólo unos pocos pudieron pugnar para convertirse en el colaborador que Horkheimer buscaba para embarcarse en el ambicioso proyecto de un libro sobre lógica dialéctica, del que se hablaba en los círculos de exiliados desde mediados de los años treinta. Tras haber barajado a candidatos como Erich Fromm, Karl Korsch o Herbert Marcuse, a comienzos de 1940 Horkheimer acabó por decantarse por Adorno. Así fue como el francfortiano se convirtió en coautor del proyecto que acabaría materializándose en el texto de Dialéctica de la llustración.

Adorno había mantenido estrecho contacto con el Instituto desde sus años en la Universidad Goethe de Fráncfort, pero sólo a partir de su llegada a Nueva York su destino individual iba a ser inseparable de él. De hecho, sólo gracias a su apoyo institucional y a la habilidad gestora de Horkheimer le fue posible cruzar el Atlántico con ciertas garantías profesionales. En realidad, la situación de Adorno como exiliado fue privilegiada, permitiéndole abandonar Europa antes del estallido de la guerra y contar con el apoyo financiero y de planificación de sus padres y del Academic Assistance Council durante su exilio en Oxford, y del Instituto a partir de su llegada a Estados Unidos. Pero no siempre fue todo tan fácil. Cuando, en 1933, los miembros del núcleo duro del Instituto estaban a salvo al otro lado de la frontera suiza, Adorno permaneció en Fráncfort, abandonado a su suerte y sin ninguna información. Sólo en octubre de 1934, con el Instituto ya instalado en Nueva York, retoma Horkheimer el contacto con él. Adorno vivió el abandono del Instituto como una traición (Adorno-Horkheimer, 2003, 19) y, en efecto, fue el único colaborador a quien no se ofreció ayuda para salir de Alemania (MüllerDoohm, 2003, 293). Pero la insistencia en la importancia de su trabajo para el Instituto no tardó en aplacar sus ánimos. El francfortiano se fue vinculando cada vez más estrechamente a Horkheimer, pasando a ser colaborador permanente del Instituto en 1935 y familiarizándose poco a poco con la idea de un futuro profesional en Estados Unidos. Tras un primer viaje a Nueva York en junio de 1937, la posibilidad de traslado definitivo llegó apenas unos meses más tarde, cuando recibió un telegrama anunciándole la oferta de participar el el Princeton Radio Research Project de la Rockefeller Foundation, dirigido por Paul Lazarsfeld (Adorno-Horkheimer, 2003, 440). La insostenibilidad de la situación en Europa, así como la posibilidad de estrechar lazos con el Instituto y colaborar con Horkheimer en proyectos que habian esbozado durante años, hizo que se disiparan sus dudas -si bien no sus reservas-, y el 26 de febrero de 1938 Adorno desembarcaba en Nueva York. Las experiencias que a partir de entonces viviria en condición de exiliado en Estados Unidos y como investigador en el proyecto dirigido por Lazarsfeld dejarían en su pensamiento una huella indeleble.

\section{Adorno y la SOCIOLOGÍA EN EL CAPITALISMO avanzado: el Princeton Radio Research Project}

Adorno llega a Estados Unidos para incorporarse al Princeton Radio Research Project, una investigación que iba a suponer un punto de inflexión en la sociología de los medios. El título exacto del proyecto era The Essential Value of Radio to all Types of Listeners, y en él se remite ya a la 
importancia que la radio había adquirido en la sociedad americana. La tecnología radiofónica se había desarrollado en Estados Unidos durante la Primera Guerra Mundial con objeto de poder mantener contacto con las tropas desplazadas a Europa, pero el comienzo de la radiodifusión no tendría lugar hasta las décadas siguientes. A partir de entonces su crecimiento fue exponencial. En apenas diecisiete años, el número familias estadounidenses con receptores radiofónicos pasa de 10.000 en 1922 a 27 millones -de un total de 32 millones de familias- en 1939 (Hullot-Kentor, 2006 , 98). En consecuencia, la radio se convierte en un instrumento privilegiado para asegurar la cohesión social, pero también para la difusión y la democratización de la cultura, especialmente de la música de la tradición clásica europea. La izquierda demócrata norteamericana consideraba que el medio radiofónico posibilitaba una redistribución de la riqueza que podía liberar a la cultura del privilegio exclusivo de las clases más pudientes y los habitantes de las grandes ciudades que hasta entonces había pesado sobre ella. En consonancia con estas expectativas, grandes emisoras como CBS o NBC aprovechaban para presentarse como "defensores del interés público y la educación" (Jenemann, 2007, 48), y se imponía la sensación de que la población estadounidense podía alcanzar por fin el "umbral de la mayoría de edad cultural" (Harper's Monthly Magazine en 1938, cit. Hullot-Kentor, 2006, 102).

\section{El Princeton Radio Research Project surge en este contexto} de grandes expectativas como una investigación sobre el valor esencial de la radio para todos sus posibles oyentes. En palabras de Hadley Cantril y Frank Stanton, ideadores del proyecto, el objetivo era determinar "el papel de la radio en la vida de diferentes tipos de oyentes, la importancia psicológica de la radio y las diferentes razones por las que a la gente le gusta escucharla" (cit. Wiggershaus, 2001, 269). En principio se trataba de una iniciativa sin precedentes en la recogida y el análisis de información sobre lo que los oyentes esperaban y valoraban de la radio, para intentar que ésta se adecuara cada vez mejor a sus expectativas. Sin embargo los datos desmienten de manera contundente toda presunta motivación altruista o interesada en la democratización de la cultura. El propósito de la investigación consistía más bien en la recogida y sistematización de datos sobre las preferencias de los oyentes para suministrárselos a una tercera parte: los sponsors, quienes podrian rentabilizar así de forma más eficiente el capital invertido. El proyecto aspiraba por tanto a la simple racionalización económica del medio radiofónico. En consecuencia, frente a la filantropía de la que presumían sus promotores, los oyentes eran considerados como meros objetos y se les tenía en cuenta a un nivel meramente administrativo. De hecho, en los años siguientes, la sociología americana se esforzó por profundizar en la senda abierta por el Princeton Radio Research Project, llegando en ocasiones a extremos de intromisión en la privacidad del oyente y planificación de la vida cotidiana sin precedentes (cfr. Jenemann, 2007, 27 y ss.). En efecto, Adorno desembarcaría en Estados Unidos para participar en una investigación pionera en la instrumentalización del conocimiento para subordinarlo a la generación de grandes beneficios empresariales, y sin duda la influencia que esta experiencia tendría en su defensa de la autonomía del pensamiento y en su crítica de la incrustación de entretenimiento e industria en los medios de masas no puede ser sobreestimada.

Tampoco puede olvidarse que Paul Lazarsfeld, director del proyecto, se iba a convertir en una de las figuras más emblemáticas en lo que a fusión de investigación académica e interés empresarial se refiere. Su habilidad más caracteristica fue siempre su capacidad para conseguir la financiación de sus investigaciones con capital de grandes empresas, y sus técnicas de medición de audiencias, que se probaron útiles para el interés empresarial, siguen estando a la base de las utilizadas hoy día. No por casualidad, en febrero de 1940, Lazarsfeld fue premiado por la industria publicitaria como el hombre que habia revelado "el valor educativo de los programas radiofónicos" (cit. Hullot-Kentor, 2006, 105). En realidad lo único que les había descubierto era el potencial económico de la radio para los posibles anunciantes. Sin embargo, en lo tocante a Adorno como director de la sección musical del proyecto, Lazarsfeld tenía expectativas especificas. Su objetivo era que la aportación del francfortiano no se limitara al fact-finding, sino que insertara sus resultados sobre el valor de la radio en la cultura americana en el marco de una teoría musical y social. Lazarsfeld le comunica explicitamente que espera de él una "aproximación europea"; es decir, "una actitud más teórica hacia el tema de investigación y una actitud más pesimista hacia el instrumento de progreso técnico" (en Adorno/Horkheimer, 2003, 482). Con ello esperaba que Adorno elevara el nivel teórico de la investigación, aportando un elemento crítico que a su vez engarzara con las expectativas del medio como democratizador de la cultura. Todo parecía indicar que el francfortiano ofrecía el perfil 
idóneo para ello. Sin embargo, si bien cuando Adorno se incorpora al Princeton Radio Research Project no contaba con ninguna experiencia previa como investigador social empírico (Adorno, 2003a, 703), lo cierto es que ya había acumulado un dilatado interés por el estudio de los medios de masas en el capitalismo avanzado ${ }^{4}$. De hecho, las primeras cartas que dirige a Lazarsfeld y el memorándum escrito en enero de 1938, todavía en Londres, revelan que Adorno se incorpora al proyecto con unos intereses de investigación ya muy definidos que iban a impedir que se plegara a lo que la dirección esperaba de él (cfr. Adorno/ Horkheimer, 2004, 427 y ss. y 503 y ss.).

Lo que más interesaba a Adorno del medio radiofónico era indagar cómo la retransmisión de la música transforma la experiencia de la misma y contribuye al cambio de su función social. Como le comunicaría a Benjamin en su última carta antes de embarcar hacia Nueva York, su inquietud de partida era que, en la estructura del broadcasting, "la música queda relegada a segundo plano" (Adorno/Benjamin, 1998, 231). Su sospecha era que, en el momento en que la música retransmitida se convertía en "música de fondo", su influencia sobre el oyente podría servir a propósitos manipuladores de forma mucho más eficiente que la retransmisión inmediata de contenidos ideológicos, y por ello pretendía estudiar de cerca la relación cuantitativa de "música, información, entretenimiento y pedagogía" (Adorno/Horkheimer, 2004, 424). Evidentemente estas motivaciones resultaban sumamente inconvenientes para los objetivos del Princeton Radio Research Project en su intento de controlar las costumbres de los oyentes y ponerlas al servicio de las empresas anunciantes, por lo que la incorporación de Adorno al proyecto tuvo poco de triunfal. Si bien la asimilación a las expectativas de Lazarsfeld podría no haberle resultado demasiado complicada, el francfortiano vivió su dependencia de éste como una amenaza a su integridad personal y teórica (cfr. Adorno/Horkheimer, 2004, 52). Por ello, desde el primer momento, se mostró obstinado en su intento de preservar su identidad intelectual y de dar continuidad al interés por la aniquilación del contenido estético del arte que había mostrado desde su primer ensayo, escrito en 1920 (Adorno, 2003f, 609), y que se había incrementado a través de los debates con Benjamin.

Ante este compromiso teórico de Adorno con la investigarción de la transformación de la función social, del contenido estético y de la experiencia de la música en el capitalismo avanzado, todos los esfuerzos de Lazarsfeld por ganar su capacidad de análisis técnico-musical para la causa de la democratización de la tradición musical europea a través de la radio estaban condenados al fracaso. No en vano el propio Adorno afirmaría que, ante el modelo de socialización total del capitalismo estadounidense, la cultura misma tenía algo de anticuado (Adorno, 2003a, 736 y s.). Por lo demás la propia formación musical y filosófica del francfortiano no era sin más asimilable a la etiqueta de "alta cultura", y se encontraba en diametral oposición a todo discurso sobre el valor pedagógico de los "bienes culturales", cuya incapacidad de dar lugar a un mundo más humano se había hecho patente en la catástrofe europea. Por ello no es de extrañar que Adorno respondiera al mayor esfuerzo pedagógico por cultivar el conocimiento y el gusto musical a cargo de la radiodifusión norteamericana, la Music Appreciation Hour de la NBC, con una de las críticas más despiadadas de toda su trayectoria (Adorno, 2006a, 247 y ss.), para escándalo de Lazarsfeld y los promotores de la investigación. $Y$ es que sin duda el francfortiano era el menos indicado para creer que la idea de formación abstracta en que se basaba dicha emisión, partidaria de grandes cánones y de la admiración de los "tesoros culturales" patrimonio de la humanidad, pudiera dar lugar a una relación viva con las obras que pretendía difundir. Dichos intentos de difusión pedagógica no conducian sino a una cosificación de la experiencia que llevaba a establecer una relación posesiva con los objetos y no ayudaban lo más mínimo a la aprehensión de su contenido. La consecuencia sería que la capacidad de escucha quedaría retenida a un nivel infantil y que todo el placer que se podía aún obtener de la música quedaba reducido al mero reconocimiento (Adorno, 1966).

Pero, si la condición de posibilidad de una verdadera democratización de la cultura debía partir del análisis de la transformación de la experiencia en la sociedad altamente industrializada, la misma regresión de la experiencia que afectaba a la recepción musical operaba en la metodología de la administrative research cultivada por Lazarsfeld. Si bien Adorno habia aterrizado en el proyecto con expectativas de poder desarrollar en él sus intereses teóricos ${ }^{6}$, su optimismo no tardaría en ser ahogado por el procedimiento de investigación al que se le exigía adaptarse. En efecto, en la metodología cuantitativa de la investigación, las preguntas de los cuestionarios establecían el marco del 
análisis y su alcance, y su cuantificación de las respuestas de los oyentes en términos meramente positivos o negativos confundian la formación cultural con el mero consumo de mercancías culturales (Adorno, 2003b, 356). Dicho procedimiento pretendía medir las reacciones individuales sin tomar en consideración cómo estaban preformadas por la propia estructura de la radio y por el conjunto de la sociedad. En consecuencia, la investigación se mostraba incapaz de cuestionar los presupuestos sociales y económicos del sistema radiofónico vigente, y mucho menos de dar cuenta de la transformación estructural de la función y la experiencia de la música en el capitalismo avanzado (Adorno, 2003a, 707 y ss. y 2003e, 298 y s.). En definitiva, a lo que el exiliado Adorno no puede resignarse en su intento de mantener cierta continuidad con sus intereses teóricos, es a que en la sociología industrializada el hombre de teoría sea sacrificado en favor del técnico de investigación (Adorno, 2003a, 714). El resultado de tal procedimiento va en detrimento tanto del conocimiento como de los propios científicos, que continúan haciendo ciencia simplemente porque se les paga, no porque tengan confianza en la relevancia 0 en el carácter vinculante de sus resultados (Adorno, 2003e,297). En su colaboración en el proyecto de Lazarsfeld, el francfortiano asiste a la pérdida de autonomía del intelectual en el proceso productivo ${ }^{7}$, y eso le refuerza en su conciencia de la necesidad de persistir en la producción teórica.

En este sentido, como correctivo a las simplificadoras contraposiciones entre Europa y Estados Unidos, se hace necesario subrayar que el positivismo y el market research con el que Adorno se topó en su llegada a Nueva York venía precisamente importado de Europa por un emigrante como Lazarsfeld, que además procedía de círculos austromarxistas ${ }^{8}$. Y es que el conflicto central en la experiencia americana de Adorno no sería el choque entre la teoría alemana en el exilio y la ciencia americana, sino más bien la capitulación de la teoría ante la ciencia organizada industrialmente (Claussen, 1999, 33) ${ }^{9}$. En su crítica a la consideración de los datos sin mediación alguna por parte de la sociología positivista, al igual que en sus intentos de desmentir la supuesta transparencia de la transmisión radiofónica, Adorno se muestra bastante menos ingenuo que los científicos al uso. Tanto la ciencia industrializada como el sistema de broadcasting presuponían la posibilidad de aislar la investigación científica y los medios de comunicación de masas de sus presupuestos tecnológicos y sociales. Frente a ello, el francfortiano argumenta que la transimisión radiofónica de una sinfonía transforma la estructura de la música transmitida tanto como la metodología de investigación positivista -con su marco de referencia "natural"- transforma sus objetos de estudio. La ilusión de transparencia ya no era posible, y los fenómenos que operaban en el medio radiofónico sólo podian entenderse si su estudio se engarzaba en el marco de una teoría de la sociedad.

Por ello, mientras que la investigación científica hegemónica se limita a proponer ajustes funcionales para mejorar el funcionamiento de la maquinaria radiofónica, Adorno se esfuerza por desarrollar una metodología fisiognómica que entendiera la radio como sintoma de toda una red de procesos sociales. El objetivo era estudiar cómo convergían en ella toda una serie de factores a la vez diversos e interdependientes: forma específica del medio, técnicas de transmisión, contenido transmitido (y cómo se transformaba en la transmisión), ilusión de cercanía y de ubicuidad, marketing, estructura empresarial de distribución y consecuencias de todo ello sobre transformación estructural de la experiencia de los contenidos y del papel del público (cfr. Adorno, 2006a, 73 y ss.). Con ello Adorno pretende resistirse a la capitulación de la teoría en el capitalismo avanzado y no resignarse al carácter superfluo que ésta estaba adquiriendo en el la industria del conocimiento. Consideraba que, por su propia condición de outsiders, los más indicados para llevar esta tarea a cabo en el pais en el que la modernidad capitalista se imponía con más fuerza eran precisamente los intelectuales exiliados (Adorno, 2003b, 355). Por eso la resistencia a la integración absoluta del emigrado era más que un asunto de mera continuidad biográfica: se trataba de una cuestión de conciencia política del intelectual.

\section{LA EXPERIENCIA AMERICANA Y EL COMPROMISO DEL INTELECTUAL}

Después de dos años de colaboración, la incompatibilidad de los planteamientos teóricos de Adorno con los objetivos del Princeton Radio Research Project se había hecho insostenible, y en junio de 1940 la financiación de su participación en la investigación se interrumpió definitivamente. A consecuencia de ello, la mayor parte del trabajo que el francfortiano había llevado a cabo en sus primeros años en 
Estados Unidos no llegaría a ver la luz o tardaría mucho en ser publicado ${ }^{10}$. De hecho, el extenso manuscrito de Currents of Music, por entonces ya prácticamente acabado, permanecería inédito hasta su edición entre los escritos póstumos en 2006. Sin embargo, las experiencias de Adorno durante estos años en Nueva York sedimentarian y acabarían por desencadenar un importante proceso de aprendizaje desde el punto de vista de su biografía intelectual. En primer lugar, el propio Adorno reconocería retrospectivamente que fue su Ilegada a Estados Unidos lo que le "desprovincializó" y le liberó de la ingenuidad de su confianza en la cultura (Adorno, 2003a, 734). En efecto, sus investigaciones en la radio le habían permitido asistir de primera mano al "entrelazamiento entre producción musical, investigación de consumo, ciencia e intereses capitalistas" (Claussen, 2006, 207), ante el cual toda concepción de alta cultura no puede sino resultar impotente. Por otra parte, durante su colaboración en el Proyecto, Adorno tuvo ocasión de familiarizarse con la metodología empírica, lo cual le "profesionalizaría" como sociólogo, pero también le permitiría, tras el regreso del Insitituto de Investigación Social a Europa, aprovechar estos conocimientos adquiridos para limpiar la sociología alemana de mala especulación e incorporar un momento empírico en la elaboración de una teoría sociológica como crítica de la sociedad.

Sin embargo, el aprendizaje decisivo que la experiencia americana iba a deparar a Adorno sería la toma de conciencia de su propia situación como filósofo y teórico de la sociedad exiliado en la era del capitalismo avanzado. Sus estudios sobre la radio y los medios de masas no le condujeron -como han pretendido sus detractores- a hacer extensible de forma aproblemática rasgos característicos del fascismo a la sociedad de consumo norteamericana, sino que le permitieron calibrar los peligros que entrañaba un modelo de sociedad que extendia una red de socialización total y trataba a los individuos como meros factores económicos a rentabilizar. Estas experiencias de Adorno coincidirian con la época en que tendrian lugar en el Instituto los principales debates entre Friedrich Pollock, Franz Neumann y Max Horkheimer sobre el carácter del capitalismo avanzado y la sociedad dirgida por monopolios, trusts y grandes acumulaciones de capital (cfr. Pollock, 1975 y Horkheimer, 1986 y 1987). El propio Adorno aportaría su parte, en 1942, con sus "Reflexiones sobre la teoría de clases", que partían de la experiencia de que "La organización total de la sociedad a través del big business y su técnica ha cubierto el mundo y su representación tan completamente, que la idea de que pudiera ser de otra forma se ha convertido casi en un afán desesperado" (Adorno, 2004, 350).

En efecto, en Estados Unidos, Adorno y el resto de exiliados del Instituto topan con una sociedad en la que impera "el capitalismo en estado puro, sin residuos precapitalistas" (Adorno, 2003a, 736) y que, precisamente por ello, ofrecía la posición más avanzada para observar las transformaciones de la civilización industrializada y las consecuencias de su proceso de racionalización. Lo decisivo de dicha experiencia se manifestaría, ya en la República Federal Alemana, en la irónica afirmación de que la relación de Europa con América se asimilaba cada vez más a la que Grecia mantuviera con el Imperio Romano. La evolución que marcaba la sociedad estadounidense revelaba que pronto tampoco quedaría ya en Europa ningún refugio al margen del capitalismo y de su modelo de socialización. De ahí la afirmación del carácter reaccionario de quien no se hubiera apropiado, de un modo u otro, de la experiencia americana (Adorno, 2003a, 737). Lo que esta experiencia revelaba era una especie de superación de la sociedad de clases sobre el mismo suelo de la sociedad de clases: "La clase dominante desaparece tras la concentración de capital. Ésta ha alcanzado una magnitud, obtenido un peso específico a través del cual se exhibe el capital como institución, como expresión de la sociedad en su conjunto" (Adorno, 2004, 353). Frente a esta concentración de poder, todo tipo de solidaridad o planteamiento de una comunidad de intereses pasaba a resultar impensable: la conformidad resultaba mucho más racional. Los estudios adornianos sobre la industria de la cultura intentaban mostrar cómo la Ilamada cultura de masas tendía a allanar el terreno en este proceso, preformando a los individuos y proporcionándoles modelos de imitación. Sin duda la situación material de las masas había mejorado y éstas estaban socialmente integradas en el sistema de producción y reproducción de la vida, pero a cambio la individualidad como tal estaba siendo sustituida por modelos de conducta producidos a escala industrial. Quien, pese a todo, no se mostraba dispuesto a adaptarse, estaba condenado a una constante impotencia. Sobre él recaía la presión de la totalidad del sistema (o del fait social durkheimiano) y se veía marcado por el estigma de la exclusión. No en vano se afirma que, dentro de semejante modelo social, ser un outsider es "la culpa más grave" (Adorno/Horkheimer, 2007, 163). 
En consecuencia, el papel de los exiliados estaba marcado de antemano. El recien llegado sólo podía justificar su presencia en Estados Unidos mediante un rendimiento social productivo. El sacrificio de la continuidad biográfica e intelectual que esto suponía para los teóricos, escritores y artistas emigrados, que a menudo implicaba incluso la renuncia a un trabajo consistente, es algo que el propio Adorno había vivido en primera persona. Sin embargo, estas experiencias dieron lugar a un proceso de concienciación de su situación personal y política que encontraría su mejor expresión en el texto de una conferencia pronunciada en 1945 ante la comunidad de exiliados californiana. En dicha intervención, titulada "Preguntas a la emigración intelectual", Adorno se dirige a los propios exiliados para intentar elaborar las experiencias comunes, e incluso intentar que, de alguna manera, dicha comunidad se convierta en sujeto político. Lo que les constituia como tal era su condición de perseguidos que habian tenido cruzar el Atlántico para sobrevivir, una identidad a la cual no podían renunciar con una entrega sin reservas a las exigencias de adaptación y de su rentabilización económica. Al fin y al cabo, que sus derechos les fueran garantizados no podía plantearse como "recompensa por un comportamiento dócil" (Adorno, 2003b, 353). Adorno llegó a hablar incluso de una "culpa del intelectual emigrado" que sacrifica su propia identidad en favor de la disciplinada sumisión al dictado de la investigación científica industrializada, y que todavía quisiera hacer de su renuncia un modelo de comportamiento moral ${ }^{11}$. Si bien el intelectual se había convertido irremisiblemente en un mero residuo en el seno de una sociedad movida por la producción industrial de conocimiento, la represión a través del consumo de productos de entretenimiento y las grandes acumulaciones de capital, esto no significaba que pudiera resignarse a perder su (relativa) autonomía. La tarea de todo intelectual exiliado consciente políticamente consistía más bien en resistirse a la seductora tentación de dejarse devorar por la industria de la cultura para entregarse a la tarea de "intentar transferir lo intransferible" (Adorno, 2003b, 357). En efecto, si el límite de la cosificación de que el proceso de racionalización socioeconómico es capaz son los propios individuos (Adorno, 2004, 364), la tarea de los últimos hombres de teoria consistirá en contribuir a desarrollar y fortalecer su resistencia a cuanto hay de inaceptable en la realidad objetiva. Y con este fin regresó el propio Adorno a Europa tan pronto como le fue posible.
Desde esta defensa de la autonomía del pensamiento, resulta significativo que el propio Instituto de Investigación Social decidiera en 1941 rechazar la oferta de fusión con la Universidad de Columbia, preservando su independencia institucional aún al precio de convertirse literalmente en outsiders del circuito académico norteamericano. Esto obligó al Instituto a reducir sus gastos y a colocar a Franz Neumann, Leo Löwenthal y Herbert Marcuse como profesores universitarios, con lo que el núcleo teórico quedó reducido a Horkheimer y el propio Adorno, que en ese mismo año se trasladaba a California para comenzar a trabajar en Dialéctica de la llustración. Cuando en 1949 el francfortiano regresa a la República Federal Alemana, vuelve reforzado por las experiencias del exilio, y pasa a convertirse en el principal exponente de la Teoría Crítica y en una voz incómoda en la autocomplacida sociedad del milagro económico que resultaría decisiva para varias generaciones de estudiantes. En estos años de posguerra, Adorno se fue haciendo consciente de que lo que había vivido como experiencia americana se estaba convirtiendo en principio universal de la sociedad globalizada. En efecto, también en Europa se imponía el capitalismo avanzado y, con él, el triunfo de la industria del conocimiento que no dejaba del Geist nada más que su cadáver (Claussen, $1999,45)$. Ante ello no queda más que persistir en la "casi irresoluble tarea" de "no dejarse atontar por el poder de los otros ni por la propia impotencia" (Adorno, 2003e, 63).

Marcado por el proceso de aprendizaje de su experiencia americana, las esperanzas adornianas se depositan en la persistencia del intelectual consecuente en una exigencia de verdad inseparable de la transformación de la sociedad que le declaraba superfluo. De un modo un tanto romántico, y de forma especialmente clara en alguno de sus últimos textos, Adorno acaba por convertir la figura del intelectual comprometido (que él mismo encarnaba) en lugarteniente de la posibilidad de transformación social a falta de un sujeto revolucionario. Su propio discípulo Hans-Jürgen Krahl señalaría lo aporético de esta necesidad de aferrarse a una subjetividad intelectual sólo capaz de articularse en academias y universidades (Krahl, 1971, 295). En efecto, si bien Adorno había esperado que la "soledad pública" del hombre de teoría en medio de crecientes concentraciones de poder llegara a ofrecer un modelo de praxis para una sociedad verdaderamente liberada, resulta imperativo constatar que, en el actual estado de las sociedades "del conocimiento" y "de la información", 
dicha soledad ya no puede siquiera aspirar a constituirse como "pública". Y sin embargo, precisamente por ello, la obra que el francfortiano concibió como un mensaje en una botella nos sigue interpelando hoy con más fuerza y urgencia que nunca. Sólo cabe esperar que su legado no caiga en el olvido.

\section{NOTAS}

1 Entre dichas recientes publicaciones destacan, sobre todo, Currents of Music. Elements of a Radio Theory (Adorno, 2006a), la edición de la correspondencia con Alban Berg (Adorno/Berg, 1997), con sus padres (Adorno, 2006b) y con Horkheimer (Adorno/Horkheimer, 2003 y 2004), así como las monografias Things Beyond Resemblance (Hullot-Kentor, 2006) y Adorno in America (Jenemann, 2007) y la biografía de Adorno a cargo de Detlev Claussen (Claussen, 2006).

2 Para las disputas en el entorno del Instituto de Investigación Social en el exilio, cfr. las monografías canónicas sobre el mismo (Jay, 1986 y Wiggershaus, 2001), así como las correspondencias de Adorno con Benjamin y con Horkheimer (Adorno-Benjamin, 1998 y Adorno-Horkheimer, 2003 y 2004), la correspondencia de Horkheimer con otros miembros del Instituto (Horkheimer 1998a y 1998b) 0 , sobre todo, la correspondencia entre Benjamin y Gershom Scholem (Benjamin-Scholem, 1987). También cabria citar malentendidos públicos como el que hubo entre Adorno y Bloch a propósito del supuesto trabajo de este último como lavaplatos (Müller-Doohm, 2003, 452-453) o las maliciosas anotaciones del diario de Bertolt Brecht en su diario sobre el Instituto (Brecht, 1973).

3 En realidad el traslado del Instituto a Ginebra ya estaba preparado antes de la toma de poder del NSDAP. Horkheimer y Pollock, director y tesorero del Instituto respectivamente, habian previsto el peligro que corrian si el nacionalsocialismo llegaba al poder. De hecho, la casa donde ambos vivían fue ocupada por la SA el mismo día de la toma de poder $y$, apenas un mes y medio después, la sede del Instituto en Fráncfort sería registrada y puesta a disposición de la Asociación de Estudiantes Nacional-Socialista (Wiggershaus, 2001, 147 y s.).

4 Además de los artículos sobre música popular que Adorno llevaba publicando desde mediados de la década de 1920 y del conocido texto sobre el jazz que se publicaría en la Zeitschrift für Sozialforschung, en 1937 Adorno había planeado también una monografía colectiva sobre la cultura de masas en la era del capitalismo monopolista. Sin embargo el proyecto no pudo llevarse a cabo por problemas de financiación (Adorno/Benjamin, 1998, 197 y 199).

5 La formación musical de Adorno estaba marcada por su militancia como miembro de la Segunda Escuela de Viena y discípulo de Alban Berg. A partir de su contacto directo con el modelo de composición de Schönberg y Berg, su interés estético se dirige a la potenciación de un modelo de cultura y de arte que combatiera la regresión de la experiencia y abriera la posibilidad de un modelo de relación sujeto-objeto no basado en el dominio. Téngase en cuenta que, ya en enero de 1926, con tan sólo veintitres años, Adorno tenía claro que su cometido teórico era "propagar lo que me parece politico-artísticamente [Kunstpolitisch] importante" (Adorno/
Aceptado: 23 de abril de 2009
ARBOR CLXXXV 739 septiembre-octubre [2009] 963-975 ISSN: 0210-1963 
Berg, 1997, 69). Sin embargo, el modelo musical con cuya difusión Adorno estaria comprometido toda su vida no sólo no se dejaba consumir desde la mera actitud contemplativa que marcaba el acercamiento estandarizado a la alta cultura burguesa, sino que se encontraba también en abierta oposición a la mayor parte de las vanguardias existentes (Adorno, 2003g).

6 En su carta del 7 de marzo de 1938, Adorno escribe a Benjamin: "El proyecto sobre la radio se ha revelado como algo con extraordinarias posibilidades y gran publicidad. Tengo la dirección de la parte musical completa $y$, además, realmente, la dirección teórica global, ya que el director oficial, Lazarsfeld, que es quien me trajo aquí, se ocupa fundamentalmente de la organización del trabajo" (Adorno/Benjamin, 1998, 234). Es posible que el tono eufórico de este pasaje quiera contribuir a disipar las dudas de Benjamin y animarle a abandonar Europa de una vez por todas y ponerse a salvo, pero las expectativas de autonomía intelectual se confirman en otros pasajes de su correspondencia (Adorno/Horkheimer, 2004, 17), y no dejan de mostrar la ingenuidad inicial de Adorno respecto del funcionamiento real de la investigación sociológica americana.

7 La consumación de este proceso en Estados Unidos no tendria lugar hasta la década de 1960, cuando la suburbanización de las ciudades y la explosión de la educación superior acabaran de reducir a los intelectuales a meros profesionales académicos acuartelados en campus departamentalizados en disciplinas de conocimiento, cada una con sus propios órganos de publicación y difusión de resultados, y todas ellas sin relación entre sí (cfr. Jacoby, 1987).
8 En 1927 Lazarsfeld había fundado en Viena un centro de investigaciones de psicología económica, en el que sentaría las bases de su metodología de investigación, y que le permitiría sobreponerse a la crisis económica de 1929 realizando por encargo los primeros análisis del mercado en Austria y un censo a gran escala de los deseos de los oyentes para la radio austriaca. El éxito de su investigación de 1930 sobre los parados de Marienthal llevó a que la Fundación Rockefeller le financiara un viaje a Estados Unidos en 1933. Sin embargo la derogación de la Constitución austriaca en 1934, la prohibición del Partido Socialista y el encarcelamiento de parte de su familia -de origen judio-, obligó a Lazarsfeld a alargar su estancia en América. En 1936 aceptó la dirección de un centro de Investigación en Newark, y allí, forzado a buscar de nuevo encargos de investigación, entró en contacto con Horkheimer y el Instituto de Investigación Social. A raíz de esta conexión, Lazarsfeld ofrecería a Horkheimer la posibilidad de que Adorno se trasladara a Estados Unidos incorporándose al Princeton Radio Research Project (Claussen, 2006, 202 y ss. y Wiggershaus, 2001, 189 y ss.).

9 En realidad, Adorno nunca fue contrario a la inclusión de un momento empírico en la sociología. A lo que se opone es a un procedimiento positivista con los datos que reduzca la teoría sociológica a la mera constatación de tautologías. Por el contrario, su posición defiende que el recurso a una base empírica bien utilizada permite reforzar el contenido de experiencia de la teoría y se convierte en una instancia de control de sus formulaciones (Adorno/Horkheimer, 2006, 427 y s.).

10 En 1941 se publican "On Popular Music" en el tomo IX de Studies on Phi- losophy and Social Science y algunos pasajes del "Analytical Study of the NBC Music Appreciation Hour" y de "The Radio Symphony" fueron incorporados más tarde en artículos hoy recogidos en El fiel correpetidor (cfr. Adorno, 2007, 169 y ss. y 379 y ss.). Por lo demás los esfuerzos de Adorno porque el gran manuscrito de Currents of Music viera la luz en Estados Unidos fueron en vano (cfr. Jenemann, 2007, 51). En general, a excepción de La personalidad autoritaria y los pocos artículos que publicó en la revista del Instituto hasta 1941, puede decirse que esta falta de reconocimiento fue el destino que pesó sobre el trabajo de Adorno durante todo el exilio, desde su colaboración con Hanns Eisler en Composición para el cine (del que retiró su nombre por la persecución política de la era McCarthy, por lo que su coautoria no se dio a conocer públicamente hasta 1969) hasta las grandes obras en que trabajó durante su estancia en Estados Unidos. Téngase en cuenta que, hasta su publicación en Ámsterdam en 1947, la única impresión de Dialéctica de la llustración era una edición casera con el título de Fragmentos filosóficos para uso interno de la comunidad de emigrados, y que Filosofía de la nueva música, Minima moralia y el Ensayo sobre Wagner sólo aparecieron después de su regreso a la República Federal Alemana. En este contexto debe entenderse que la célebre metáfora del mensaje en una botella expresaba también la urgencia de Adorno por conseguir que su obra trascendiera más allá del círculo de exiliados alemanes. $Y$ esto es algo que sólo lograría tras su regreso a Europa.

11 "El intelectual que, sin embargo, obedece la fórmula del 'así es como 
se hace aqui', se comporta de modo similar a aquellos judios en Alemania de los que decía el chiste que habian participado en el día del Partido [del NSDAP] en Nuremberg llevando carteles en los que se leía 'Fuera con nosotros.' 'Dentro con nosotros' es lo mismo, pero visto desde la otra parte" (Adorno, 2003b, 353).

\section{BIBLIOGRAFÍA}

Adorno, Theodor W. (1962): "Aldous Huxley y la utopía", en Prismas. La crítica de la cultura y la sociedad, Barcelona, Ariel, pp. 99-125.

Adorno, Theodor W. (1966): "Sobre el carácter fetichista en la música y la regresión del oído", en Disonancias. Música en el mundo dirigido, Madrid, Rialp, pp. 17-70.

Adorno, Theodor W. (2003a): "Wissenschaftliche Erfahrungen in Amerika", en Gesammelte Schriften 10.2, Fráncfort del Meno, Suhrkamp, pp. 702740.

Adorno, Theodor W. (2003b): "Fragen an die intellektuelle Emigration", en Gesammelte Schriften 20.1, Fráncfort del Meno, Suhrkamp, pp. 352-358.

Adorno, Theodor W. (2003c): "Über Tradition", en Gesammelte Schriften 10.1, Fráncfort del Meno, Suhrkamp, pp. 310-320.

Adorno, Theodor W. (2003d): "El curioso realista", en Notas sobre literatura. Obra completa 11, Tres Cantos, Akal, pp. 372-392.

Adorno, Theodor W. (2003e): Minima Moralia. Reflexionen aus dem beschädigten Leben, Gesammelte Schriften 4, Fráncfort del Meno, Suhrkamp.

Adorno, Theodor W. (2003f): "El expresionismo y la veracidad artística", en Notas sobre literatura. Obra completa 11, Tres Cantos, Akal, pp. 589-591.
Adorno, Theodor W. (2003g): "Die Stabilisierte Musik", en Gesammelte Schriften 18, Fráncfort del Meno, Suhrkamp, pp. 721-727.

Adorno, Theodor W. (2004): "Reflexiones sobre la teoría de clases", en Escritos sociológicos I. Obra completa 8, Tres Cantos, Akal, pp. 357-364.

Adorno, Theodor W. (2005): TraumprotokoIle, Fráncfort del Meno, Suhrkamp.

Adorno, Theodor W. (2006a): Currents of Music. Elements of a Radio Theory, Fráncfort del Meno, Suhrkamp.

Adorno, Theodor W. (2006b): Cartas a los padres, Buenos Aires, Paidós.

Adorno, Theodor W. (2007): Composición para el cine. El fiel correpetidor, Obra completa 15, Tres Cantos, Akal.

Adorno, Theodor W. y Benjamin, Walter (1998): Correspondencia 1928-1940, Madrid, Trotta.

Adorno, Theodor W. y Berg, Alban (1997): Briefwechsel 1925-1935, Fráncfort del Meno, Suhrkamp.

Adorno, Theodor W. y Horkheimer, Max (2003): Briefwechsel I: 1927-1937, Fráncfort del Meno, Suhrkamp.

Adorno, Theodor W. y Horkheimer, Max (2004): Briefwechsel II: 1938-1944, Fráncfort del Meno, Suhrkamp.

Adorno, Theodor W. y Horkheimer, Max (2007): Dialéctica de la Ilustración. Obra completa 3, Tres Cantos, Akal.

Benjamin, Walter y Scholem, Gershom (1987): Correspondencia 1933-1940, Madrid, Taurus.

Brecht, Bertolt (1973): Arbeitsjournal, Fráncfort del Meno, Suhrkamp [2 vols.].

Claussen, Detlev (1999): "Die amerikanische Erfahrung der kritischen Theoretiker", en Claussen, Detlev, Negt, Oskar y Werz, Michael (eds.) (1999), Keine Kritische Theorie ohne Amerika. Hannoversche Schriften I, Fráncfort del Meno, Neue Kritik, pp. 27-45.

Claussen, Detlev (2006): Theodor W. Adorno. Uno de los últimos genios, Va- lencia, Publicaciones Universidad de Valencia.

Erdmann, Karl Dietrich (1989): Deutschland unter der Herrschaft des $\mathrm{Na}$ tionalsozialismus, Múnich, Deutsche Taschenbuch Verlag.

Horkheimer, Max (1987): "Autoritärer Staat", en Gesammelte Schriften 5, Fráncfort del Meno, Fischer, pp. 293-319.

Horkheimer, Max (1988): "Die Juden und Europa", en Gesammelte Schriften 4, Fráncfort del Meno, Fischer, pp. 308331.

Horkheiemer, Max (1998a): Gesammelte Schriften 16. Briefwechsel 1937-1940, Fráncfort del Meno, Fischer.

Horkheiemer, Max (1998b): Gesammelte Schriften 17. Briefwechsel 1941-1948, Fráncfort del Meno, Fischer.

Hullot-Kentor, Robert (2006): "Second salvage. Prolegomenon to a Reconstruction of Currents of Music", en Things Beyond Resemblance. Collected Essays on Theodor W. Adorno, Nueva York, Columbia University Press, pp. 94-125.

Jacoby, Russell (1987): The Last Intellectuals. American Culture in the Age of Academy, Nueva York, Basic Books.

Jay, Martin (1986): La imaginación dialéctica. Una historia de la Escuela de Fráncfort, Madrid, Taurus.

Jenemann, David (2007): Adorno in America, Minnesota, University of Minnesota Press.

Krahl, Hans-Jürgen (1971): Konstitution und Klassenkampf, Fráncfort del Meno, Neue Kritik.

Müller-Doohm, Stefan (2003): En tierra de nadie. Theodor W. Adorno, una biografía intelectual, Barcelona, Herder.

Pollock, Friedrich (1975): "Staatskapitalismus", en Stadien des Kapitalismus, Múnich, Beck, pp. 72-100.

Wiggershaus, Rolf (2001): Die Frankfurter Schule. Geschichte, theoretische Entwicklung, politische Bedeutung, Múnich, Deutsche Taschenbuch Verlag. 